\title{
Epitaxial Growth and Characterization of Zinc-blende CrAs/GaAs/MnAs/GaAs Multilayers
}

\author{
W. H. Wang*, T. Manago, and H. Akinaga \\ Research Consortium for Synthetic Nano-Function Materials Project (SYNAF), Nanotechnology Research Institute (NRI), \\ National Institute of Advanced Industrial Science and Technology (AIST), 1-1-1 Umezono, Tsukuba, Ibaraki 305-8568, Japan
}

(Received 14 November 2005)

\begin{abstract}
We report on the growth, structural and transport properties of zinc-blende CrAs/GaAs/MnAs/GaAs multilayers on GaAs(001) substrates. Structural analyses using in-situ reflection high-energy electron diffraction and $e x$ situ cross-sectional transmission electron microscopy confirmed the realization of a zinc-blende crystal structure. Room temperature Hall measurements reveal that the as-grown multilayer exhibits p-type conductivity with a very low resistivity of $0.052 \Omega \mathrm{cm}$, a high carrier concentration of $6.2 \times 10^{19} \mathrm{~cm}^{-3}$ and a Hall mobility of $1.8 \mathrm{~cm}^{2} / \mathrm{Vs}$. We also observed a clear decrease of the resistivity in samples after low temperature annealing.
\end{abstract}

Key words : half-metallic ferromagnets, zinc-blende multilayer, hall effect

\section{Introduction}

Half-metallic ferromagnets are expected as a key ingredient in future high performance spintronic devices, because they have only one electronic spin channel at the Fermi energy and, therefore, show nearly $100 \%$ spin polarization [1,2]. Since de Groot et al.'s discovery [3] in 1983, many kinds of half-metallic ferromagnets have been theoretically predicted and some of them, such as Heusler alloys [4,5], $\mathrm{CrO}_{2}[6]$, and transition-metal perovskites [7] have been confirmed experimentally. On the other hand, it is highly desirable to grow half-metallic ferromagnetic thin films on semiconductors because of the possibility of an application to "spintronic" devices. For this purpose, considerable efforts have been made on the epitaxial growth of the transition-metal pnictides, such as MnAs [7], $\mathrm{MnSb}[8,9]$, and $\mathrm{MnBi}$ [10] on GaAs substrates.

Using the full-potential linearized augmented-planewave (FLAPW) method, Akinaga et al. [11] first calculated that in the ferromagnetic case CrAs with a zincblende structure could be a half-metallic ferromagnet. Following the prediction, they have succeeded to grow thin films of CrAs on $\mathrm{GaAs}(001)$ substrates with the atomically flat interfaces by molecular-beam epitaxy [11].

*Corresponding author: Tel: +81-29-861-5080 (Ext. 55531), Fax: +81-29-861-3211, e-mail: wang.wenhong@aist.go.jp
They found that CrAs is ferromagnetic at room temperature with the Curie temperature higher than $400 \mathrm{~K}$ and they have deduced a total spin-moment of $3 \mu_{B}$ in agreement with the theory. Nevertheless, the very thin critical thickness $(3 \mathrm{~nm}$ ) of zinc-blende CrAs film is one of the biggest challenges faced in advancing this material toward applications. More recently, $a b$ initio calculations indicated the half-metallic properties are preserved in zinc-blende CrAs/GaAs hybrid multilayers [12]. Experimentally, zinc-blende $\mathrm{CrAs} / \mathrm{GaAs}$ multilayers up to $20 \mathrm{~nm}$ have been successful grown by molecular-beam epitaxy [13], but with a very high resistivity and low carrier concentration. Theoretical calculation predicted that $\mathrm{Mn}$ dopant substitutes for the Ga site and acts as an acceptor providing itinerant holes [14]. For this reason, we assume that the existence of MnAs layer may result in the increase of the carrier concentration in $\mathrm{CrAs} / \mathrm{GaAs}$ system. Therefore, in this paper, we report on the growth, structural and transport properties of zinc-blende $\mathrm{CrAs} / \mathrm{GaAs} /$ $\mathrm{MnAs} / \mathrm{GaAs}$ hybrid multilayer on $\mathrm{GaAs}(001)$ substrates.

\section{Experimental Details}

A molecular-beam-epitaxy (MBE) system (Riber 32P) was used to grow zinc-blende $\mathrm{CrAs} / \mathrm{GaAs} / \mathrm{MnAs} / \mathrm{GaAs}$ multilayer. Figure 1 shows the schematic structure of the multilayer study in this work. The multilayer was grown on an "epi-ready" semi-insulating GaAs(001) substrate. 


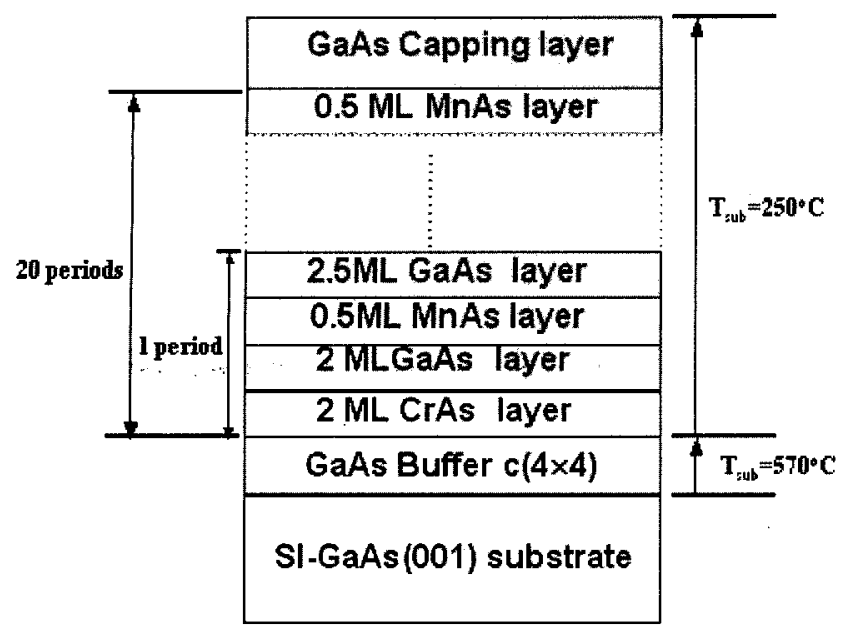

Fig. 1. A cross-sectional schematic drawing of the CrAs/ $\mathrm{GaAs} / \mathrm{MnAs} / \mathrm{GaAs}$ multilayer.

The substrate was set on a molybdenum holder with indium solder. After being degassed at $400^{\circ} \mathrm{C}$ in a loading chamber, the substrate was transferred into the MBE growth chamber without breaking vacuum. Before starting the growth, the substrate was heated up to about $590^{\circ} \mathrm{C}$ and kept for 10 minutes for thermal cleaning to remove the surface oxidization layer. Then, a GaAs buffer layer with the thickness of $20 \mathrm{~nm}$ was grown at $570^{\circ} \mathrm{C}$ to prepare a flat surface. On top of the buffer layer, 2 monolayers (ML) of CrAs, $2 \mathrm{ML}$ of GaAs, $0.5 \mathrm{ML}$ of MnAs and $2.5 \mathrm{ML}$ of GaAs were grown alternatively at $250^{\circ} \mathrm{C}$. Growth of the CrAs, GaAs, and MnAs layers was performed by the opening of Knudsen cells for each element of $\mathrm{Cr}, \mathrm{Ga}$ and $\mathrm{Mn}$ under exposure of As with a beam pressure of about $4 \times 10^{-6} \mathrm{~Pa}$. Finally, a GaAs capping layer with a thickness of $10 \mathrm{~nm}$ was deposited to prevent oxidation of the multilayer. The surface was characterized by in-situ reflection high-energy electron diffraction (RHEED) during the growth. The structural properties were examined by ex-situ cross-sectional transmission electron microscopy (TEM). Transport measurements were carried out at room temperature in Hall-bars fabricated by photolithography and wet etching.

\section{Results and Discussion}

Figure 2 shows the RHEED patterns of the GaAs surface and the last MnAs layer of the multilayer sample with electron azimuth along the [110] and [110] axes of GaAs, respectively. For the GaAs substrate, the RHEED patterns [Figs. 2(a) and 2(b)] were taken at $570^{\circ} \mathrm{C}$ after the growth of $20 \mathrm{~nm} \mathrm{GaAs}$ buffer layer. We note that along the $[1 \overline{1} 0]$ direction the reconstruction shows four lines in comparison to two lines along [110], indicating a
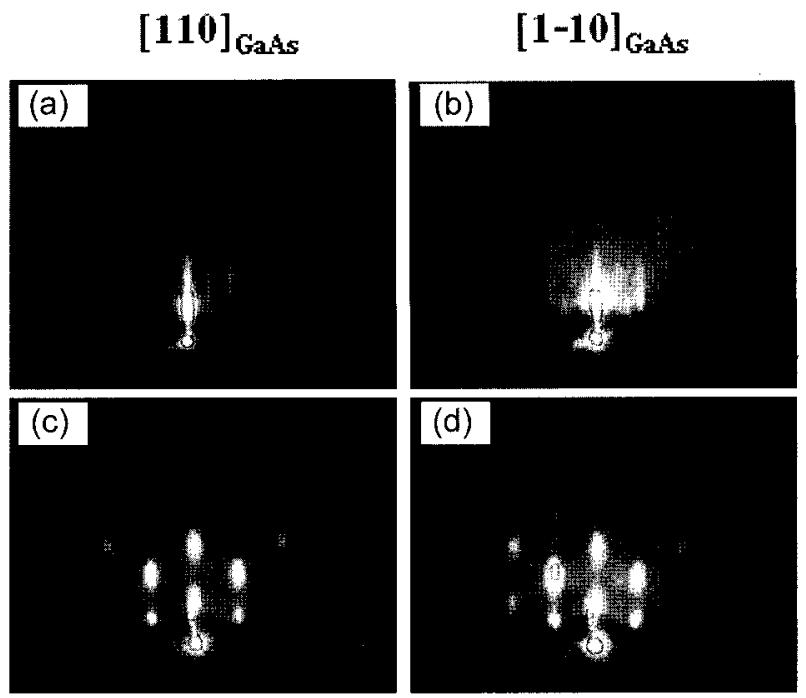

Fig. 2. RHEED patterns from the surface of (a)-(b) $20 \mathrm{~nm}$ GaAs buffer layer and (c)-(d) the last MnAs layer for the [110] azimuth (left-hand side) and the [1-10] azimuth (right-hand side).

smooth (2×4)-reconstructed $\mathrm{GaAs}(001)$ surface. Upon cooling, the $(2 \times 4)$-reconstructed surface changes to "As rich" c (4×4)-reconstructed surface at around $520^{\circ} \mathrm{C}$ and As shutter was closed below $400^{\circ} \mathrm{C}$. The GaAs substrate retains the c $(4 \times 4)$-reconstructed surface before the deposition of the initial CrAs layer at $250^{\circ} \mathrm{C}$. Soon after the deposition of $1 \mathrm{st} \mathrm{CrAs}$ layer at $250^{\circ} \mathrm{C}$, we found that the reconstruction lines in the substrate's RHEED patterns disappear immediately, and the $\mathrm{c}(4 \times 4)$ RHEED pattern changes to the $(1 \times 1)$ one. Importantly, the principal streaks lines remained during several repetitions of the alternate growth, which suggests the success of an epitaxial growth of the $\mathrm{CrAs} / \mathrm{GaAs} / \mathrm{MnAs} / \mathrm{GaAs}$ multilayer. Moreover, we found that the streaky pattern gradually changed to slightly spotty pattern with the increase of the periods, and an obvious spotty pattern was observed when the last growth period was performed. Figure 2(c) and (d) show the RHEED patterns of the last MnAs layer surface of the multilayer.

The structural properties of the multilayer were further checked by high-resolution TEM. Figure 3(a) shows a representative wide-view cross-sectional TEM image of the zinc-blende multilayer. We can see that the multilayer was grown nearly as a planned structure because the thickness of $\sim 20 \mathrm{~nm}$ was very close to the designed value of $23 \mathrm{~nm}$. Figure 3(b) shows an enlarged interface zone of the multilayer. It can be clearly observed that the lattice image of multilayer forms continuous lines with the GaAs substrate, which indicated that $\mathrm{CrAs}$, GaAs and MnAs composing the multilayer maintain zinc-blende structure. 


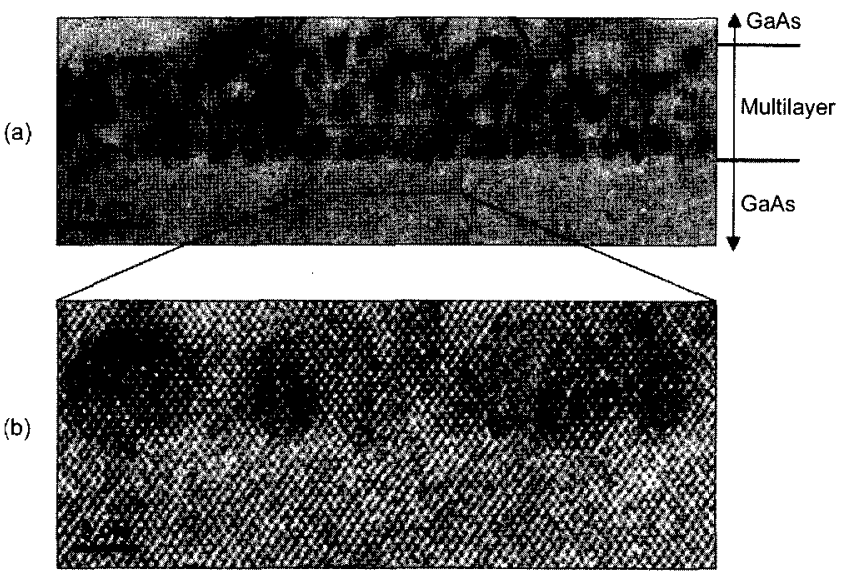

Fig. 3. (a) Cross-sectional TEM image of the multilayer with the structure of $[\mathrm{CrAs}(2 \mathrm{ML}) / \mathrm{GaAs}(2 \mathrm{ML}) / \mathrm{MnAs}(0.5 \mathrm{ML}) /$ $\operatorname{GaAs}(2.5 \mathrm{ML})] \times 20$ layers, grown on a $20 \mathrm{~nm}$ GaAs buffer layer and capped by a $10 \mathrm{~nm}$ GaAs layer. (b) An enlarged interface zone between GaAs substrate and the multilayer.

On the other hand, however, it should be pointed out that a lot of tiny clusters (zinc-blend type) with around $2 \sim 3$ $\mathrm{nm}$ in diameter are observed in the whole multilayer region, as shown in our TEM images. Formation of defects and clusters have been observed in highly mismatched structures like InAs/GaAs where the lattice mismatch is $7.2 \%$, and have been explained by the relaxation of the lattice strain [15]. The lattice mismatch between the hypothetical zinc-blende MnAs and GaAs is predicted to be around $6.0 \%$. Therefore, we assume that lattice mismatch between zinc-blend MnAs and GaAs resulted in the formation of defects/clusters in our hybrid multilayer. In fact, gradually spotty RHEED patterns observed during MBE growth of the multilayer in our present work supporting above assumption. It means even for $0.5 \mathrm{ML}$ MnAs layer, lattice strain relaxation has induced tiny clusters. Therefore, our ongoing works are to maximize the concentration of $\mathrm{Mn}$ substitutionally incorporated in the cation site of GaAs to act as acceptors rather than as compensating interstitial defects or clusters.

Hall effect and electrical resistivity measurements were carried out at room temperature in a Hall-bar fabricated by photolithography and wet etching. The results are summarized in Table 1. The as-grown multilayer exhibits p-type conductivity with a very low resistitivity of 0.052 $\Omega \mathrm{cm}$, a high carrier concentration of $6.2 \times 10^{19} \mathrm{~cm}^{-3}$ and a mobility of $1.8 \mathrm{~cm}^{2} / \mathrm{Vs}$ at room temperature. Moreover, ex situ postgrowth low-temperature annealing (at $200^{\circ} \mathrm{C}$ for 1 hour) was also performed to reduce the interstitial defect density. The results of Hall measurements for the annealing sample are also summarized in Table 1. We can see that, as expect, the resistivity decreases and the carrier
Table 1. Transport properties at room temperature of [CrAs $(2 \mathrm{ML}) / \mathrm{GaAs}(2 \mathrm{ML}) / \mathrm{MnAs}(0.5 \mathrm{ML}) / \mathrm{GaAs}(2.5 \mathrm{ML})] \times 20$ multilayers.

\begin{tabular}{ccccc}
\hline \hline Sample & Type & $\begin{array}{c}\text { Mobility } \\
\left(\mathrm{cm}^{2} / \mathrm{Vs}\right)\end{array}$ & $\begin{array}{c}\text { Resistivity } \\
(\Omega \mathrm{cm})\end{array}$ & $\begin{array}{c}\text { Carrier density } \\
\left(\mathrm{cm}^{-3}\right)\end{array}$ \\
\hline As-grown & $\mathrm{p}$ & 1.81 & 0.052 & $6.2 \times 10^{19}$ \\
Anneal $\left(\right.$ at $\left.200^{\circ} \mathrm{C}\right)$ & $\mathrm{p}$ & 1.82 & 0.042 & $6.5 \times 10^{19}$ \\
\hline
\end{tabular}

concentration increases in sample after low-temperature annealing, although the mobility remain almost unchanged.

\section{Conclusion}

We have demonstrated the successful epitaxial growth of CrAs (2ML)/GaAs(2ML)/MnAs(0.5ML)/GaAs(2.5ML) multilayer on GaAs(001) substrates by MBE. In-situ RHEED patterns during the growth and ex-situ crosssectional TEM images confirmed the realization of a zincblende crystal structure. Room temperature Hall measurements reveal that the as-grown multilayer exhibits $p$-type conductivity with a very low resistivity of $0.052 \Omega \mathrm{cm}$, a high carrier concentration of $6.2 \times 10^{19} \mathrm{~cm}^{-3}$ and a Hall mobility of $1.8 \mathrm{~cm}^{2} / \mathrm{Vs}$. The resistivity decreases clearly in samples after low temperature annealing.

\section{Acknowledgements}

This work was partly supported by the New Energy and Industrial Technology Development Organization (NEDO). A part of this work has also conducted in AIST-NanoProcessing Facility, supported by "Nanotechnology Support Project" of the Ministry of Education, culture, Sports, Science and Technology (MEXT), Japan. The authors are grateful to Dr. Fumiyoshi Takano and Dr. Sinsarp Asawin for helpful discussion, Mr. Ikezu for technical assistance for MBE growth. One of the authors (W. H. Wang.) would like to acknowledge Japan Society for the Promotion of Science (JSPS) for fellowship grant (JSPS Id: P05611).

\section{References}

[1] R. A. Groot, F. M. Mueller, P. G. van Engen, and K. H. J. Buschow, Phys. Rev. Lett. 502024 (1983).

[2] G. A. Prinz, Phys. Today 48, 58 (1995).

[3] T. Ambrose, J. J. Krebs, and G. A. Prinz, Apply. Phys. Lett. 76, 3280 (2000).

[4] W. Van Roy, J. De Boeck, B. Brijs, and G. Borghs, Apply. Phys. Lett. 77, 4190 (2000). 
[5] W. H. Wang, M. Przybylski, W. Kuch, L. I. Chelaru, J. Wang, Y. F. Lu, J. Barthel, H. L. Meyerheim, and J. Kirschner, Phy. Rev. B 71, 144416 (2005).

[6] N. E. Brener, J. M. Tyler, J. Callaway, D. Bagayoko, and G. L. Zhao, Phys. Rev. B 61, 16582 (2000).

[7] J. Z. Sun, W. J allagher, P. R. Duncombe, L. KrusinElbaum R. A.Altma, A. Gupta, Yu. Lu, G. Q. Gong, and G. Xiao, Appl. Phys. Lett. 69, 3266 (1996).

[8] S. Miyanishi, H. Akinaga, W. van Roy, K. Tanaka, App. Phys. Lett. 70, 2046 (1997).

[9] M. Mizuguchi, H. Akinaga, K. Ono, and M. Oshima, Appl. Phys. Lett. 76, 1743 (2000).
[10] R. Hayashi, K. Terayama, T. Ishibashi, and K. Sato, J. Magn. Soc. Japan 23, 99 (1999).

[11] H. Akinaga, T. Manago, and M. Shirai, Japan. J. Appl. Phys. 39, L1118 (2000).

[12] C. Y. Fong and M. C. Qian, J. Phys.: Condens. Matter 16, S5669 (2004).

[13] H. Akinaga and M. Mizuguchi, J. Phys.: Condens. Matter 16, S5549 (2004).

[14] T. Dietl et al., Science 287, 1019 (2000).

[15] S. Dosanjh, P. Dawson, M.R. Fahy, B. A. Joyce, T. Murry, H. Toyoshima, X. M. Zhang, and R. A. Stradling, J. Appl. Phys. 71, 1242 (1992). 